mathematical papers, where the case of the collision of equivalent particle-observers is fully dealt with. You cannot wantonly re-graduate any casual clock and expect to secure a consistent physics. Kinematical relativity does not do so. In kinematical relativity it is shown that for consistent time-keeping to be possible, the various particle-observers whose tem. poral experiences constitute clocks must be members of equivalences. Regraduation is applied only to such equivalences; and it was shown by Whitrow and myself $^{2}$ that if two members of an equivalence ever coincide, then all members coincide at the same event. This technical point is well known to all who have studied time-keeping in relation to equivalences; it is fundamental, for example, in some recent unpublished work by A. G. Walker. In its application, it means that in a contracting universe of time-keepers, there would be a singularity which would be the counterpart of 'creation' in an expanding universe. Prof. Dingle is concerned with the timing of 'subsequent events'; there would be no subsequent events-Prof. Dingle would not survive the catastrophe-just as in an expanding universe there are no events anterior to 'creation'. There are other objections to a contracting universe, but the 'absurdity' which preoccupies Prof. Dingle is a monster of his own construction which simply adds point to the absurdity of a contracting universe. As the universe is observed to be expanding, Prof. Dingle's difficulties never arise.

Prof. Dingle appears to resent my referring him to my published papers. I did so because I resent being continually required to re-traverse in the looseness of verbal statement ground carefully covered, with all due reservations, in the technical papers. Prof. Dingle has recently suggested that kinematic relativity cannot account for the red-shift in the spectra of the galaxies, whereas kinematical relativity is essentially the exploration of the properties of a cloud of mutually receding particles. If Prof. Dingle does not like clock-regraduation, let him content himself with the $t$-scale, which describes a consistent physics as it stands; only, he will have difficulty with reconeiling its results with Newtonian mechanics unless he faces the fact that Newtonian mechanics employs the $\tau$-scale, not the $t$-scale.

Wadham College,

E. A. Mrnne.

Oxford. Oct. 15.

${ }^{1}$ Nature, 158, 389 (1945).

2 Z. Astrophys., 15, 277 (1938).

Prop. Milne states that I have misapplied his prineiple of elock-regraduation. If so, Prof. Haldane has made the same mistake. The lamp-post example was his, and his letter ${ }^{1}$ expressed his belief that Milne's transformation was applicable to it. The only difference between us was that he trusted that Milne could make his transformation "lead to the same predictions of observable events"-he stated that this could be done-and I did not. Now Prof. Milne fails him by denying the obligation.

Nevertheless, Haldane and I are right. In Milne's accounts of his theory (in "Relativity, Gravitation and World-Structure", for example) the right to regraduate clocks is first claimed on a priori grounds independent of applications. Only afterwards are equivalences defined as consisting of observers who impose a particular voluntary restriction on this general right. Now Prof. Milne wants to deny the right to all but these observers. On what grounds ? Indeed, for that matter, are not the nose and lamppost members of an equivalence? They can fulfil the conditions, and no minimum number of actual observers is preseribed. Prof. Milne can, of course, claim that he can make what arbitrary distinctions he likes in an ideal hypothesis: in that case I challenge his right to call a romance a scientific theory. If, on the other hand, he says that the system of nebulæ provides an application for the theory, then I ask why the student of nebulæ may regraduate his clock, but not the student of lampposts or meteorites.

Imperial College, S.W.7.

Herbert Dingle.

${ }^{2}$ Nature, 156, 266 (1945).

\section{Clock-Regraduations and Relativistic Cosmology}

Prof. E. A. Mrune has severely criticized relativistic cosmology for several reasons, one being that this theory is restricted to a definite time-scale, whereas his own theory admits of an infinite number of time-scales. Milne's construction of an 'equivalence' is invariant over a clock-regraduation, that is, a change from one time-scale to another.

In $1943^{1}$, I suggested that the true analogue of clock-regraduations in relativity theory was to be found in Weyl's transformation of gauge. For the metrics of relativistic cosmology, this reduces to a change of the function $\gamma$ in the line-element,

$$
d s^{2}=\gamma(r, t) \cdot\left(d t^{2}-d x^{2}-d y^{2}-d z^{2}\right) .
$$

It follows as a corollary to Infeld's ${ }^{2}$ remarks that, with this interpretation, Maxwell's equations and Dirac's equations are invariant over clock-regraduations, while the relations of spherical symmetry and homogeneity are also preserved.

These remarks seem to dispose of one, at least, of Milne's main criticisms of relativistic cosmology.

Balloon Development Establishment, T. J. WrLLMORE. Cardington, Bedford. Aug. 18.

1 Willmore, T. J., Ph.D. Thesis (London, 1943).

'Infeld, L., Nature, 156, 112 (1945).

\section{Adsorption Isotherms from Chromato- graphic Measurements}

DE VAULT ${ }^{1}$ has directed attention to the possibility of obtaining the adsorption isotherm of single solutes from chromatographic results of the 'diffuse' boundary, suggesting a differential equation for evaluating the measurements. A more useful equation can be obtained by integration of the equation

$$
v / x-\alpha=d q / d c,
$$

where $\alpha$ and $q$ are the pore space and the amount adsorbed per gram of adsorbent. After developing with a given amount of pure solvent $v$, the amount of solute $\mu$ remaining in the column between the tail end of the chromatogram and the point $\bar{x}$ where the concentration $\bar{c}$ exists is

$$
[\mu] \bar{c}=\int_{c=0}^{c=\bar{c}}(q+\alpha c) d x \text {. . . . }
$$

